\title{
Projector Model for Efficient List-Mode Reconstruction in PET Scanners with Parallel Planar Detectors
}

\author{
Juan E. Ortuño, Giancarlo Sportelli, Member, IEEE, Pedro Guerra, Member, IEEE, and \\ Andrés Santos, Senior Member, IEEE
}

\begin{abstract}
We have developed a new projector model specifically tailored for fast list-mode tomographic reconstructions in Positron emission tomography (PET) scanners with parallel planar detectors. The model provides an accurate estimation of the probability distribution of coincidence events defined by pairs of scintillating crystals. This distribution is parameterized with 2D elliptical Gaussian functions defined in planes perpendicular to the main axis of the tube of response (TOR). The parameters of these Gaussian functions have been obtained by fitting Monte Carlo simulations that include positron range, acolinearity of gamma rays, as well as detector attenuation and scatter effects. The proposed model has been applied efficiently to list-mode reconstruction algorithms. Evaluation with Monte Carlo simulations over a rotating high resolution PET scanner indicates that this model allows to obtain better recovery to noise ratio in OSEM (ordered-subsets, expectationmaximization) reconstruction, if compared to list-mode reconstruction with symmetric circular Gaussian TOR model, and histogram-based OSEM with precalculated system matrix using Monte Carlo simulated models and symmetries.
\end{abstract}

\section{INTRODUCTION}

System response accuracy has been demonstrated to play a $S_{\text {crucial factor to improve the image quality in iterative }}$ statistical reconstruction methods applied to PET. Due to the high data dimensionality in 3D-PET, tremendous efforts have been done to respect realistic models without incurring in excessive computational costs [1].

Monte Carlo simulation has been widely used to estimate accurately the system response for data projection without the inherent complexity of analytical models [2-4]. For this purpose, validated PET simulations tools like GATE [5] or PeneloPET [6] have been used. Another possibility to estimate the system matrix is to use real point-source measurements in combination with numerical fitting to analytical models $[7,8]$.
However, both Monte Carlo results and analytical estimations must be precomputed off-line and stored as a system matrix, whose size can require enormous storage for 3D-PET imaging. The size reduction provided by histogram compression, symmetries [9], quasi-symmetries [10], axial mashing [11] and factorization as a product of sparse matrices [12] can greatly reduce the need for memory [8], but if the number of histogram bins required to guarantee the intrinsic system resolution is greater than the number of events, listmode reconstruction becomes a good alternative for fast and accurate reconstruction. Moreover, the introduction of additional information as time of flight, depth of interaction and energy of detected photons can involve compromising the data accuracy with the precalculated system matrix approach [13].

In list-mode reconstruction, the system matrix factorization scheme can only be performed in the image space and not in the projection space. Thus, factorization imposes accuracy limits in modeling projection-dependent blurring effects [14]. In this work, we present a projector model for list-mode reconstruction, applicable to PET scanners composed of parallel planar detectors, that provides a system response with accuracy comparable to Monte Carlo simulated, with the additional advantage of being numerically computable on-thefly.

\section{MATERIALS AND METHODS}

\section{A. Monte Carlo Simulation}

Several representative TORs were simulated with Monte Carlo methods and the result was fitted to the proposed analytical functions to obtain the kernel models. The simulated TORs were chosen to be contained between the minimum and maximum crystal difference between a pair of opposed planar detectors.

To improve simulation statistics, both the positron generation region and direction of annihilated gamma rays is limited to reduce close-to-zero possibilities of detection in the selected pair of crystals. This leads to select a cylinder of radius $r$ oriented to the center of the pair of scintillating crystals as region of positron generation, and a maximum $\theta$ for the azimuthal angle of emission, as is shown in Fig. 1. 

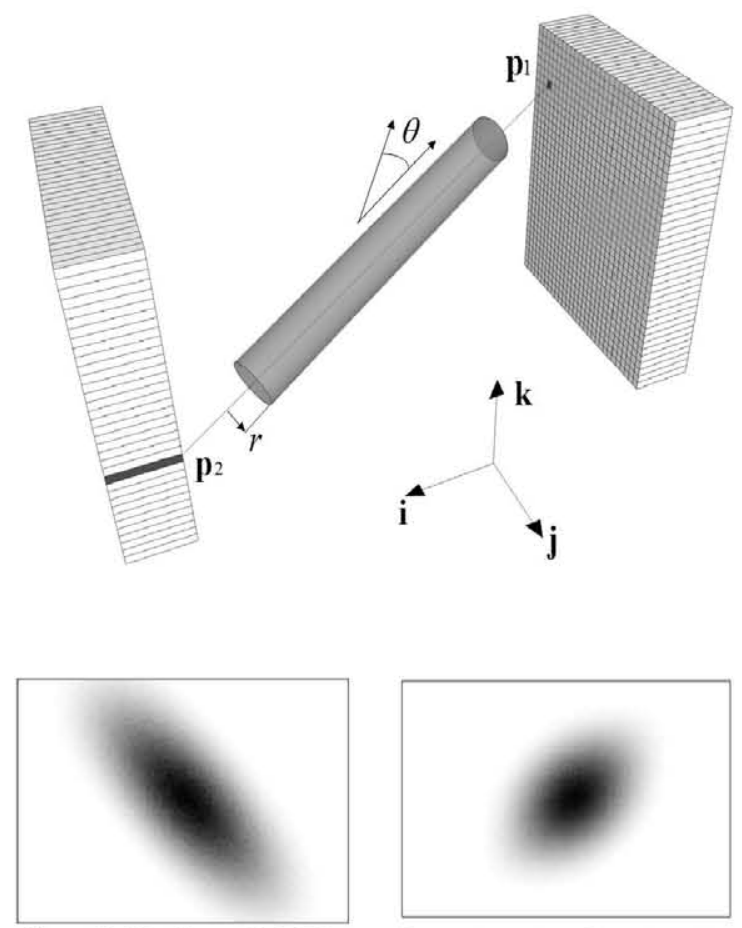

$C_{1}=(3,3), C_{2}=(26,31)$

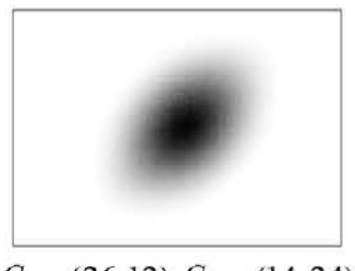

Fig. 1. Up: Scheme of a cylindrical region centered in the LOR where the positrons are generated during the Monte Carlo simulation; down: Two Examples of 2D Elliptical Gaussians histograms of probability distributions of a TOR between pairs of scintillating crystals $\mathrm{c}_{1}-\mathrm{c}_{2}: \mathrm{c}_{1}=(3,3)$ with $\mathrm{c}_{2}=(26,31)$ and $\mathrm{c}_{1}=(26,12)$ with $\mathrm{c}_{2}=(14,24)$, where the two indexes indicates the associated column and row of the scintillating crystal.

Monte Carlo code models the gamma ray acollinearity, positron range, as well as scintillating crystal attenuation and scatter effects. All physical effects are simulated with respect to a water medium, which approximates the majority of biological tissues, and $18^{\mathrm{F}}$ sources. Geometrical and detector characteristics of the small animal rPET scanner [15] were used in the Monte Carlo modeling.

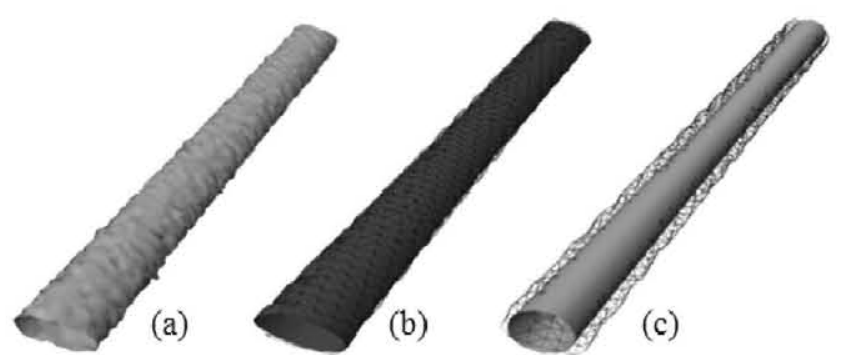

Fig. 2.(a) Isosurface of Monte Carlo simulation of an oblique TOR; (b) Elliptical Gaussian model (red) with superimposed simulation (line grid); (c) Circular Gaussian model (cyan) with superimposed simulation (line grid).

Monte Carlo simulation is also used to determine the most probable points of detection for every pair of scintillating crystals, which define the ideal LOR. These points were selected to be $5 \mathrm{~mm}$ off the detector surface.

\section{B. Projector Kernel}

Monte Carlo simulations show that the system response is approximately shaped as an elliptical tube with a 2D Gaussian distributed section:

$$
p(x, y)=A \exp \left(-\frac{x^{2}}{2 \sigma_{x}^{2}}-\frac{y^{2}}{2 \sigma_{y}^{2}}\right)
$$

The projector kernel, i.e. the scalar field that describes the TOR, has been derived with respect to a reference system in which the $\mathrm{z}$ axis is aligned with the ideal line of response, (LOR) while $\mathrm{x}$ and $\mathrm{y}$ axes are aligned with detectors. For any LOR, a 3D transformation converts the coordinates of every voxel center $\mathbf{p}=\mathbf{x} \mathbf{i}+\mathbf{y} \mathbf{j}+\mathbf{z k}$ on the new reference frame $\mathbf{p}^{\prime}=$ $x^{\prime} \mathbf{i}^{\prime}+y^{\prime} \mathbf{j} \mathbf{y}^{\prime}+z^{\prime} \mathbf{k}$ '. The transformation can be expressed in homogeneous coordinates as a rotation matrix $\mathbf{R}$ and a translation vector $\mathbf{t}$ :

$$
\left[\begin{array}{l}
\mathbf{p}^{\prime} \\
1
\end{array}\right]=\left[\begin{array}{ll}
\mathbf{R} & \mathbf{t} \\
\mathbf{0}^{T} & 1
\end{array}\right]\left[\begin{array}{l}
\mathbf{p} \\
1
\end{array}\right]
$$

Vectors of $\mathbf{R}$ are normal to the plane perpendicular to the LOR (denoted as $\mathbf{r}_{3}$ ), the plane that contains the LOR and the principal axis of the pixelated crystals (denoted as $\mathbf{r}_{2}$ ) and the plane perpendicular to $\mathbf{r}_{2}$ and $\mathbf{r}_{3}$ (denoted as $\mathbf{r}_{1}$ ); $\mathbf{p}_{1}$ and $\mathbf{p}_{2}$ are the vertices of the LOR and $\mathbf{n}$ is the unit vector normal to the detector; $\mathbf{p}_{0}$ Is a generic point belonging to the LOR:

$$
\begin{gathered}
\mathbf{R}=\left[\begin{array}{c}
\mathbf{r}_{1}^{T} \\
\mathbf{r}_{2}^{T} \\
\mathbf{r}_{3}^{T}
\end{array}\right], \quad \mathbf{t}=-\mathbf{R} \mathbf{p}_{0} \\
\mathbf{r}_{3}=\frac{\mathbf{p}_{2}-\mathbf{p}_{1}}{\left\|\mathbf{p}_{2}-\mathbf{p}_{1}\right\|}, \quad \mathbf{r}_{2}=\frac{\mathbf{r}_{3} \times \mathbf{n}}{\left\|\mathbf{r}_{3} \times \mathbf{n}\right\|}, \quad \mathbf{r}_{1}=\mathbf{r}_{2} \times \mathbf{r}_{3}
\end{gathered}
$$

Fig. 1 shows two examples of the simulated elliptical Gaussian sections corresponding to TORs between two selected pairs of scintillating crystals $\mathrm{c}_{1}-\mathrm{c}_{2}: \mathrm{c}_{1}=(3,3)$ with $c_{2}=(26,31)$ and $c_{1}=(26,12)$ with $c_{2}=(14,24)$, where the two indexes indicates the column and row of the scintillating crystal, respectively. The complete isosurface of a simulated TOR is sketched in Fig. 2, along with the fitted 2D elliptical Gaussian model and a standard circular Gaussian model.

The complete set of simulated TORs projected over the plane $z^{\prime}=0$ were fitted to elliptical Gaussian functions. In Fig.3, values of $\sigma_{\mathrm{x}}$ and $\sigma_{\mathrm{y}}$ are plotted against the distance between main crystals axes. It can be observed that $\sigma_{\mathrm{x}}$ values increases with the total difference of crystals associated to the modeled LOR, while $\sigma_{\mathrm{y}}$ remain constant. A polynomial fit was used to approximate the crystal differences not modeled with the Monte Carlo method. 


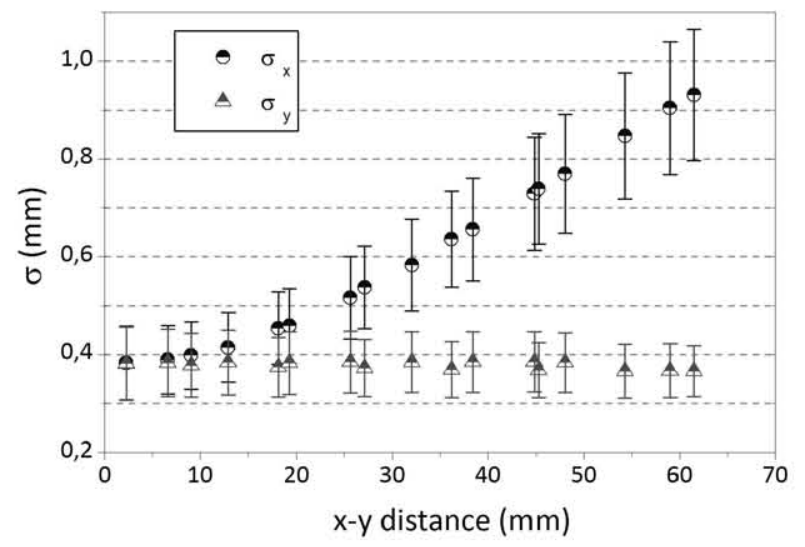

Fig.3. Fitted values of standard deviations of elliptical Gaussians for representative TORs in Monte Carlo simulations. TOR skewness is expressed as distance between associated pixelated crystals in the $x-y$ detector plane.

\section{Reconstruction algorithm}

The list-mode 3D-OSEM reconstruction algorithm used to test the proposed kernel model is based on the algorithm described in [16]. It can be summarized as:

$$
\lambda_{j}^{k+1}=\frac{\lambda_{j}^{k}}{s_{j}} \sum_{i \in L_{n}} \frac{a_{i j}}{\sum_{j^{\prime}} a_{i j^{\prime}} \lambda_{j^{\prime}}^{k}}
$$

All observed coincidence events, or LORs, are divided into $N$ subsets $L_{n}$; index $k$ is equal to $k=N l+n$, being $n$ the subset number and $l$ the total passes through the whole dataset (i.e., the number of iterations); $\lambda_{j}^{k}$ is the intensity estimation for the
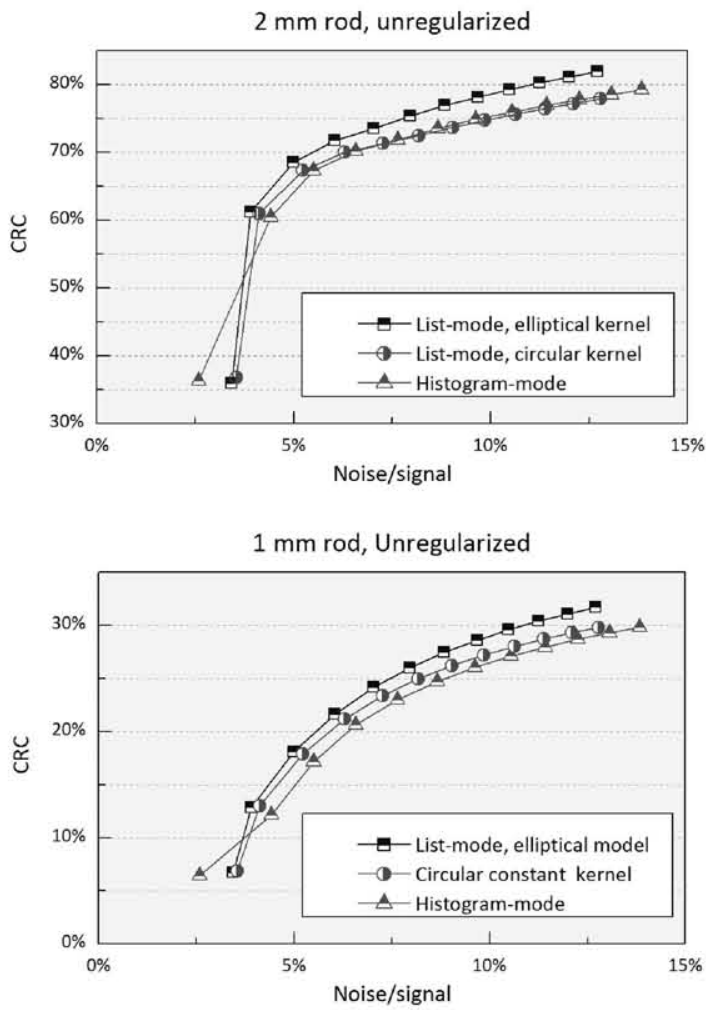

voxel $j$ at sub-iteration $k, s$ is the sensitivity image, and $a_{i j}$ is the likelihood of an observed event $i$ for a given voxel $j$. Scatter and random estimates have been neglected. In order to prevent reconstruction biases, list-mode subsets have been composed by sampling uniformly the whole acquired dataset with offsets $\mathrm{n}$ belonging to $[0, \mathrm{~N}-1]$.

\section{RESULTS}

The proposed kernel has been integrated in an efficient listmode OSEM algorithm [17]. Reconstructed data have been compared with histogram-mode 3D-OSEM [18] with precalculated system-matrix using Monte Carlo methods and listmode OSEM with symmetric Gaussian model [17]. Direct (i.e., unregularized), and Median Root Prior (MRP) regularized versions [19] of the reconstruction algorithms were used. The simulation package GATE has been used to describe the rPET scanner [15] and simulate projection data for the NEMA phantom. Ten subsets were used in all cases.

Contrast recovery coefficient (CRC) to noise/signal ratios until 120 iterations have been reported in Fig. 4. The measurements were done with an Image Quality Phantom defined according to the NEMA NU4-2008 standard for small animal PET (reconstructed in Fig. 5) over $1 \mathrm{~mm}$ and $2 \mathrm{~mm}$ hot rods. Results show that the proposed formulation outperforms both histogram-mode 3D-OSEM with precalculated system matrix and list-mode 3D-OSEM with symmetrical Gaussian TOR model.
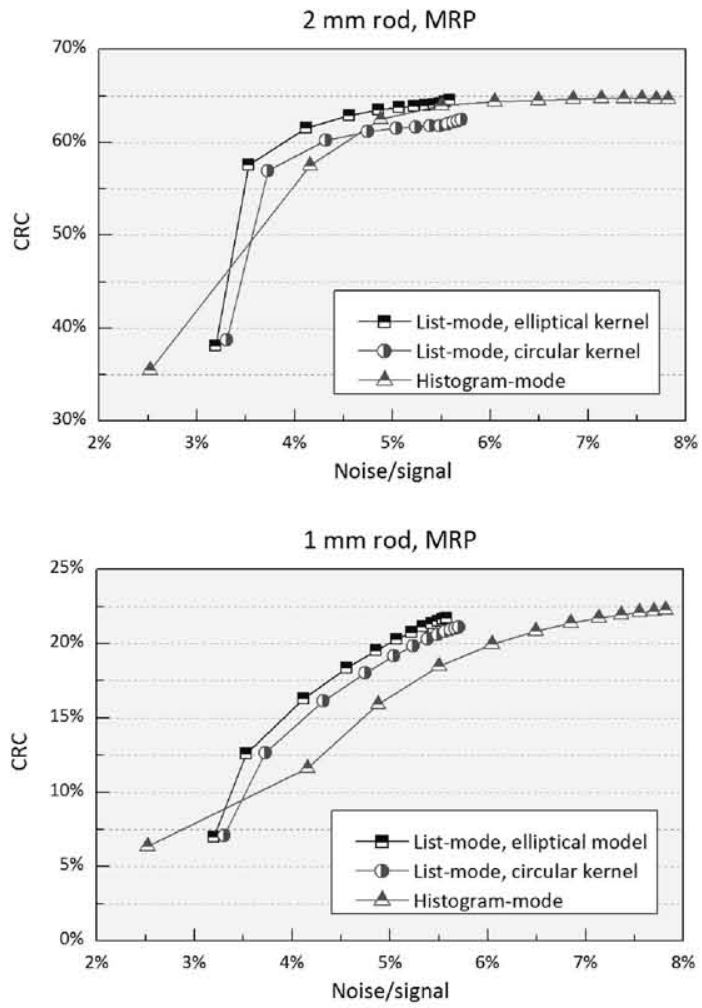

Fig. 4. Recovery contrast coefficient (CRC) vs. noise to signal ratio measurements over $1 \mathrm{~mm}$ and $2 \mathrm{~mm}$ rods of the NEMA quality phantom. 


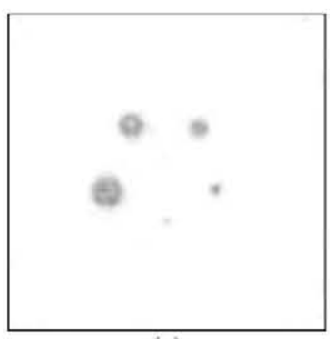

(a)

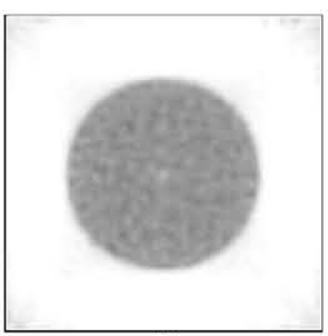

(b)

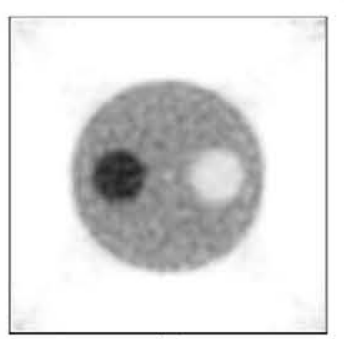

(c)

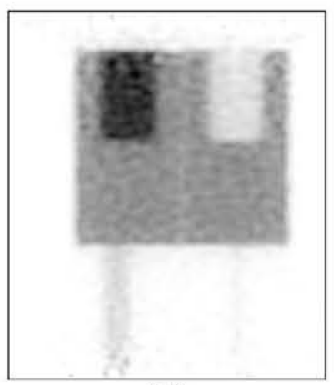

(d)

Fig. 5. Transaxial and axial sections of the NEMA quality phantom reconstructed with the elliptical Gaussian model and list-mode OSEM.

\section{CONCLUSIONS}

We have simulated and developed a new method for LOR projection suitable for fast list-mode reconstructions. The method provides a methodology to construct a look-up table of parameter and a numerical formulation for the a posteriori probability distribution over the field of view (i.e., standard deviations of 2D oriented Gaussian kernels). Numerical values are obtained by fitting Monte Carlo simulations which include crystal attenuation and scatter effects. It has been shown that with the given formulation it is possible to improve reconstruction quality figures with respect to system matrix based histogram mode and list-mode 3D-OSEM reconstructions based on symmetrical Gaussian tubes of response.

\section{REFERENCES}

[1] C. S. Levin, "New imaging technologies to enhance the molecular sensitivity of positron emission tomography," Proceedings of the IEEE, vol. 96, (3), pp. 439-467, 2008.

[2] E. N. Gimenez, E. Nacher, M. Gimenez, J. M. Benlloch, and M. Rafecas, "Comparison of different approaches based on Monte Carlo methods to calculate the system matrix for small animal PET," Nuclear Instruments \& Methods in Physics Research Section a-Accelerators Spectrometers Detectors and Associated Equipment, vol. 569, (2), pp. 346-349, 2006.

[3] M. Rafecas, B. Mosler, M. Dietz, M. Pogl, A. Stamatakis, D. P. McElroy, and S. I. Ziegler, "Use of a Monte Carlo-based probability matrix for 3-D iterative reconstruction of MADPET-II data," IEEE Transactions on Nuclear Science, vol. 51, (5), pp. 2597-2605, 2004.

[4] L. Zhang, S. Staelens, R. Van Holen, J. De Beenhouwer, J. Verhaeghe, I. Kawrakow, and S. Vandenberghe, "Fast and memory-efficient Monte Carlo-based image reconstruction for whole-body PET," Medical Physics, vol. 37, (7), pp. 3667-3676, 2010.

[5] S. Jan, G. Santin, D. Strul, S. Staelens, K. Assie, D. Autret, et. al., "GATE: a simulation toolkit for PET and SPECT," Physics in Medicine and Biology, vol. 49, (19), pp. 4543-4561, 2004.

[6] S. Espana, J. L. Herraiz, E. Vicente, J. J. Vaquero, M. Desco, and J. M. Udias, "PeneloPET, a Monte Carlo PET simulation tool based on PENELOPE: features and validation," Physics in Medicine and Biology, vol. 54, (6), pp. 1723-1742, 2009.
[7] V. Y. Panin, F. Kehren, C. Michel, and M. Casey, "Fully 3-D PET reconstruction with system matrix derived from point source measurements," IEEE Transactions on Medical Imaging, vol. 25, (7), pp. 907-921, 2006.

[8] S. Stute, D. Benoit, A. Martineau, N. S. Rehfeld, and I. Buvat, "A method for accurate modelling of the crystal response function at a crystal sub-level applied to PET reconstruction," Physics in Medicine and Biology, vol. 56, (3), pp. 793-809, 2011.

[9] C. A. Johnson, Y. C. Yan, R. E. Carson, R. L. Martino, and M. E. Daube-Witherspoon, "A system for the 3D reconstruction of retractedsepta PET data using the EM algorithm," IEEE Transactions on Nuclear Science, vol. 42, (4), pp. 1223-1227, 1995.

[10] J. L. Herraiz, S. Espana, J. J. Vaquero, M. Desco, and J. M. Udias, "FIRST: Fast Iterative Reconstruction Software for (PET) tomography," Physics in Medicine and Biology, vol. 51, (18), pp. 4547-4565, 2006.

[11] S. Vandenberghe, M. E. Daube-Witherspoon, R. M. Lewitt, and J. S. Karp, "Fast reconstruction of 3D time-of-flight PET data by axial rebinning and transverse mashing," Physics in Medicine and Biology, vol. 51, (6), pp. 1603-1621, 2006.

[12] J. Y. Qi, R. M. Leahy, S. R. Cherry, A. F. Chatziioannou, and T. H. Farquhar, "High-resolution 3D Bayesian image reconstruction using the microPET small-animal scanner," Physics in Medicine and Biology, vol. 43, (4), pp. 1001-1013, 1998.

[13] A. J. Reader, "The promise of new PET image reconstruction," Physica Medica, vol. 24, (2), pp. 49-56, 2008.

[14] C. Cloquet, F. C. Sureau, M. Defrise, G. Van Simaeys, N. Trotta, and S. Goldman, "Non-Gaussian space-variant resolution modelling for listmode reconstruction," Physics in Medicine and Biology, vol. 55, (17), pp. 5045-5066, 2010.

[15] J. J. Vaquero, E. Lage, L. Ricon, M. Abella, E. Vicente, and M. Desco, "rPET detectors design and data processing," in IEEE Nuclear Science Symposium Conference Record, New York, 2005, pp. 2885-2889.

[16] A. J. Reader, R. Manavaki, S. Zhao, P. J. Julyan, D. L. Hastings, and J. Zweit, "Accelerated list-mode EM algorithm," IEEE Transactions on Nuclear Science, vol. 49, (1), pp. 42-49, 2002.

[17] G. Sportelli, J. E. Ortuño, and A. Santos, "Efficient Rendering of Regions of Response in List-Mode Reconstruction for PET," in this IEEE Nuclear Science Symposium Conference Record, 2011.

[18] J. E. Ortuño, G. Kontaxakis, J. L. Rubio, P. Guerra, and A. Santos, "Efficient methodologies for system matrix modelling in iterative image reconstruction for rotating high-resolution PET," Physics in Medicine and Biology, vol. 55, (7), pp. 1833-1861, 2010.

[19] G. Kontaxakis, L. G. Strauss, T. Thireou, M. J. Ledesma-Carbayo, A. Santos, S. A. Pavlopoulos, and A. Dimitrakopoulou-Strauss, "Iterative Image Reconstruction for Clinical PET Using Ordered Subsets, Median Root Prior and Web a Based Interface," Medical Imaging and Biology, vol. 4, (3), pp. 219-231, 2002. 